\title{
Compresión medular por linfoma no Hodgkin difuso. Descripción de caso y revisión de literatura
}

\author{
Spinal cord compression due to diffuse non-Hodgkin's lymphoma. Case \\ description and literature review
}

Compressão da medula espinhal devido ao linfoma difuso não-Hodgkin. Descrição do caso

\author{
e revisão de literatura
}

\section{Cristina Carolina Canchignia Mayorga (1) 1, Luis Antonio Mazón López (1) 1, Gabriela Isabel Núñez Matamoros 구 1, Jennifer Nathaly Delgado Carreño ${ }^{10}{ }^{1}$, Washington Javier Ladines Castro (iD) ${ }^{2}$}

\begin{abstract}
1 Postgrado de Medicina Interna, Hospital Clínica San Francisco, Guayaquil - Ecuador; ${ }^{2}$ Servicio de Hematología, Hospital Clínica San Francisco, Guayaquil - Ecuador.

Correspondencia a:

Cristina Carolina Canchignia Mayorga,

cristy_camy@hotmail.com
\end{abstract}

Recibido: 18 de mayo, 2021

Aceptado: 10 de septiembre, 2021 Publicado: 18 de noviembre, 2021

\section{CASO CLÍNICO}

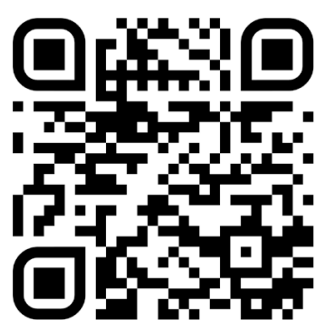

Escanea en tu dispositivo móvil o revisa este artículo en: https:// revistaclinicaguayaquil.org

\section{RESUMEN}

El linfoma difuso de células grandes tipo $\mathrm{B}$ es el más común de los linfomas no Hodgkinianos (30-35\%). Su curso clínico y pronóstico es variable, y depende de la gran heterogeneidad genética o subtipos, lo que sugiere una etiología muy variada (factores del huésped, infecciosos, etc). Más del $50 \%$ de los pacientes son mayores de 60 años, y constituye una enfermedad de rápido o muy rápido crecimiento con clínica que depende de la localización tumoral. Se presenta caso clínico de un paciente con inicio súbito de dolor abdominal y afectación neurológica en relación a nivel de compresión medular.

Palabras clave: Linfoma no Hodgkin; Linfoma difuso de células B grandes; Paraplejia

\section{ABSTRACT}

Diffuse large B-cell lymphoma constitutes the most common group of the non-Hodgkin's lymphomas (30-35\%). Its clinical course and prognosis are variable, and depend on its genetic heterogeneity or subtypes, which suggests a highly variable etiology (host factors, infectious causes, etc.). More than $50 \%$ of patients are older than 60 years, and it constitutes a fast or very fast-growing disease with symptoms that depend on the tumor location. We present a case of a patient with sudden onset of abdominal pain and neurological involvement in relation to the level of spinal compression.

Key words: Non-Hodgkin Lymphoma; Diffuse large B-cell lymphoma; Paraplejia

\section{RESUMO}

A pancreatite hipertrigliceridêmica aguda (PATG) é descrita como causa O linfoma difuso de grandes células B é o linfoma não-Hodgkin mais comum(30-35\%), seu curso clínico e prognóstico évariável, dependendo da grande heterogeneidade genética ou subtipos, sugerindo uma grande variedade de etiologia (fatores hospedeiros, infecciosos, etc.). Mais de $50 \%$ dos pacientes com mais de 60 anos de idade, é uma doença de crescimento rápido ou muito rápido com sintomas clínicos que dependem da localização do tumor. Apresentamos um caso de um paciente com início súbito de dor abdominal e envolvimento neurológico em relação ao nível de compressão da medula espinhal.

Palavras-chave: Linfoma não-Hodgkin; Linfoma difuso de grandes células B; Paraplegia 


\section{INTRODUCCIÓN}

El linfoma difuso de células $B$ grandes es el subtipo más frecuente de neoplasias de células B maduras (30-35\%), que usualmente se presenta en la séptima década de la vida (1). Surge en el mediastino, a partir de las células B tímicas en la etapa terminal de diferenciación con características morfológicas, inmunohistoquímicas, genotípicas, y clínicas distintivas (2). Representa el $80 \%$ de los linfomas agresivos con alto grado de malignidad y de crecimiento rápido, lo que permite su diagnóstico en etapas iniciales (I-II) de la clasificación de Ann Arbor; por esta razón el compromiso de la medula ósea no es frecuente.

A pesar de la dificultad del diagnóstico extraganglionar dada su infrecuente presentación, los estudios histológicos e inmunohistoquímicos son efectivos para evitar un diagnóstico erróneo (3). El diagnóstico se basa exclusivamente en estudios de inmunohistoquímica y genéticos que se realizan en tejidos tomados mediante una biopsia tumoral (1). La terapéutica se determina de acuerdo con el estadio, biología molecular y factores de riesgo asociados; siendo R-CHOP (rituximab, ciclofosfamida, clorhidrato de doxorubicina (hidroxidaunorubicina), sulfato de vincristina y prednisona), el esquema más usado como primera línea, con alto nivel de evidencia (2).

\section{REPORTE DE CASO CLÍNICO}

Paciente masculino de 67 años con antecedentes patológicos de hipertensión arterial controlado con losartan y atenolol, enfermedad cerebrovascular isquémica sin secuela neurológica y portador de marcapasos unicameral por bloqueo auriculoventricular. Acude al hospital con cuadro clínico de dolor abdominal difuso, disnea, acompañado de deposiciones líquidas con relajación de esfínteres, dorso lumbalgia y paraparesia en las últimas 24 horas. Al ingreso el paciente se encontraba afebril, con buen estado general, a la exploración abdominal sin hallazgos relevantes, a la exploración neurológica llama la atención paraparesia 1/5 flácida, arrefléctica, con nivel sensitivo T2-T3, reflejos cutáneos abdominales abolidos e hipoestesia distal en calcetín.

En los estudios paraclínicos se evidencia LDH de $1657 \mathrm{U} / \mathrm{L}$, en el electrocardiograma un bloqueo de rama izquierda, y en el ecocardiograma se diagnosticó miocardiopatía dilatada con fracción de eyección ventricular del $50 \%$. Los estudios de extensión por imagen demostraron una masa pleural tipo bulky $(12 \times 7.5 \times 6 \mathrm{~cm})$ con conglomerados ganglionares paraaórticos, paravertebrales, supra e infra diafragmáticos e inguinales derechos, con daño de estructura ósea e infiltración espinal (Figura 1).

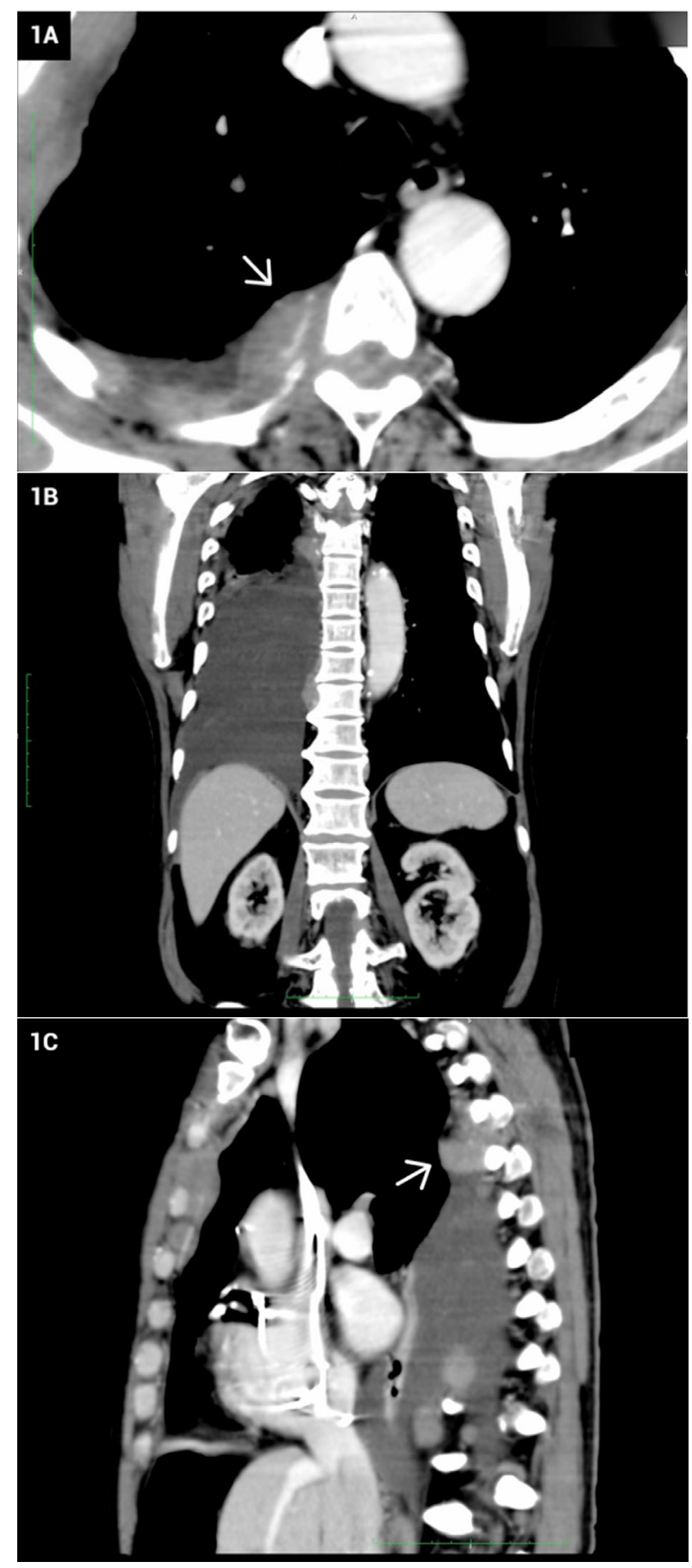

Figura 1. A. Corte axial, masa paravertebral derecha adyacente a T5-76 que mide aproximadamente $3 \mathrm{~cm}$, impresiona extensión hacia canal medular. B. Corte coronal, masa paravertebral derecha adyacente a T9-710-T11, mide aproximadamente $3 \mathrm{~cm}$. C. Corte sagital, masa paravertebral que comprime e infiltra canal medular. 


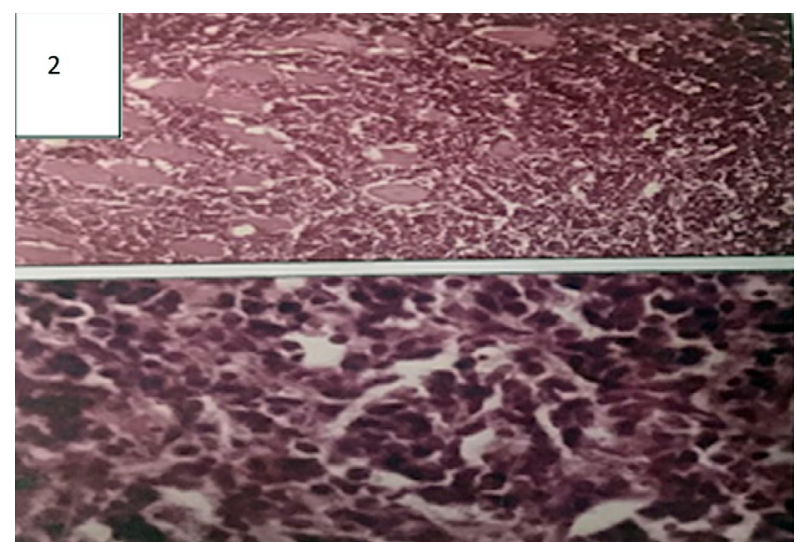

Figura 2. Corte histológico que muestra la estructura de tejidos blandos, infiltrados extensamente por neoplasia maligna de patrón sólido, constituida por proliferación difusa de células pequeñas, redondas y azules de apariencia linfocitaria, cuyos núcleos son hipercromáticos y con escaso citoplasma, las mismas que infiltran las fibras musculares estriadas y a tejido conectivo fibroso. Las células neoplásicas son positivas para marcadores linfoides (CD45) y marcadores linfoides de fenotipo $B(C D 20)$ con negatividad para linfocitos $\mathrm{T}(\mathrm{CD} 3)$.

Se realizó biopsia excisional por medio de toracotomía más laminectomía con exéresis del tumor, más descompresión del canal medular. El reporte histopatológico reveló neoplasia maligna de patrón sólido con inmunohistoquímica con CD20 positivo compatible con Linfoma no Hodgkin difuso de células grandes inmunofenotipo B (Figura 2), con clasificación IV de Ann Arbor, estadio funcional ECOG 4, con IPI 3 puntos, conformando parte del grupo de riesgo intermedio-alto, con una tasa de supervivencia global a los 5 años del $43 \%$.

Con respecto al déficit neurológico en el postoperatorio mediato, la fuerza muscular de extremidades pélvicas se recuperó $4 / 5$, con mejoría de la sensibilidad, posterior al inicio de quimioterapia de primera línea con esquema R-CHOP, cuya dosis del antracíclico se ajustó al $50 \%$ debido a su patología cardiovascular; sin embargo, cursó con evolución desfavorable, además, se asoció un proceso infeccioso pulmonar, siendo derivado a la unidad de cuidados intensivos con requerimiento de asistencia respiratoria mecánica y posterior fallecimiento.

\section{DISCUSIÓN}

El compromiso extranodal en los linfomas no Hodgkinianos (LNH) afecta habitualmente al tubo digestivo, piel, médula ósea y el sistema nervioso central 6. La afectación del espacio epidural por linfoma representa del 10 al 30\% de las neoplasias malignas epidurales, siendo estos los LNH de alto grado de malignidad como en el caso que se describe (4), su presentación clínica se relaciona a la afectación local en relación al segmento de columna vertebral afectada y a la compresión medular en dicho nivel (5).

Patogénicamente estos linfomas se inician en tejidos blandos paraespinales bajo la forma de una adenomegalia paravertebral que secundariamente invade la médula espinal a través del foramen vertebral, sin destruir ni infiltrar tejido óseo (6), afectando el espacio epidural a partir de la extensión de la masa paravertebral o lesión vertebral (7). El segmento dorsal de la columna se afecta con mayor frecuencia, seguido de la columna cervical y lumbar (6). El diagnóstico diferencial, debido a la infiltración epidural, debe realizarse con carcinomas metastásicos, sarcomas y abscesos epidurales de distinta etiología (4).

La descompresión quirúrgica puede mejorar el déficit neurológico en los casos de compromiso extranodal (7), como en el caso del paciente presentado; otros autores no recomiendan el abordaje quirúrgico de esta clase de pacientes por la alta mortalidad asociada a la cirugía.

Sutratamientoquimioterapéuticoseindividualiza de acuerdo con el grado de la enfermedad (5), de los factores de riesgo asociados y del estado de salud del enfermo previo al diagnóstico; sin embargo, el tratamiento con el esquema R-CHOP continúa siendo el gold standard.

\section{CONCLUSIÓN}

El caso corresponde a una presentación clínica poco habitual, a nivel extranodal con invasión de columna medular espinal del linfoma difuso de células B grandes; es fundamental el abordaje diagnóstico precoz para establecer un estadiaje y tratamiento oportuno de esta patología, considerando los marcadores pronósticos y comorbilidades de cada paciente.

A pesar del tratamiento, el paciente descrito se encontraba en un estadio avanzado, con pronóstico desfavorable, cuyo descenlace fue fatal, demostrando así la alta agresividad y tasa de morbimortalidad en este tipo de presentaciones de LNH.

\section{REFERENCIAS BIBLIOGRÁFICAS}

1. Caballero Barrigón MD, Martín A. Linfoma difuso de células B grandes. En: Molins de Rei, editor. Manual práctico de Hematología Clínica. 5a ed. Barcelona: Antares; 2015. p. 349-58. 
2. Peñalver F-J, Sancho J-M, de la Fuente A, Olave M-T, Martín A, Panizo C, et al. Guidelines for diagnosis, prevention and management of central nervous system involvement in diffuse large B-cell lymphoma patients by the Spanish Lymphoma Group (GELTAMO). Haematologica. 2017;102(2):235-45. DOI:10.3324/haematol.2016.149120

3. Lenz G, Staudt LM. Aggressive lymphomas. $N$ Engl J Med. 2010;362(15):1417-29. DOI:10.1056/NEJMra0807082

4. Simiele Narvarte A, Gómez Rodríguez N, Novoa Sanjurjo F. Compromiso epidural espinal como presentación de los linfomas no hodgkinianos: aportación de 6 casos. An Med Interna. 2003;20(9):28-31.

5. Sciubba D, Witham T, Gokaslan Z. Management of metastasic spine disease with spinal cord compression. Neurosurg Quart. 2007;17(1):40-7.

6. Corti M, Carolis L De, Centioni A, Velásquez J, Rizzo C, Narbaitz M. Síndrome de compresión medular como forma de presentación de linfoma no Hodgkin epidural espinal primario. Descripción de un caso y revisión de la literatura. Neurol Argentina. 2017;9(2):1204. DOI:10.1016/j.neuarg.2016.04.006

7. Evans LS, Hancock BW. Non-Hodgkin lymphoma. Lancet. 2003;362(9378):139-46. DOI:10.1016/S0140-6736(03)13868-8

\section{ACERCA DE LOS AUTORES}

1. Cristina Carolina Canchignia Mayorga. Postgrado de Medicina Interna, Hospital Clínica San Francisco, Guayaquil - Ecuador.

\section{ORCID: 0000-0002-9100-7640}

2. Luis Antonio Mazón López. Postgrado de Medicina Interna, Hospital Clínica San Francisco, Guayaquil - Ecuador.

ORCID: 0000-0002-8939-2797

3. Gabriela Isabel Núñez Matamoros. Postgrado de Medicina Interna, Hospital Clínica San Francisco, Guayaquil Ecuador.

ORCID: 0000-0002-3150-694X

4. Jennifer Nathaly Delgado Carreño. Postgrado de Medicina Interna, Hospital Clínica San Francisco, Guayaquil Ecuador

ORCID: 0000-0001-7008-3778

5. Washington Javier Ladines Castro. Servicio de Hematología, Hospital Clínica San Francisco, Guayaquil - Ecuador

ORCID: 0000-0001-9012-2180 